Problems relating to the Impact of Waves on a Spherical Obstacle in an Elastic Medium. By Prof. Horack LaMrb, F.R.S. Read and received March 8th, 1900.

The scattering of elastic waves by a spherical obstacle whose properties differ slightly from those of the surrounding medium was discussed long ago, and applied to the illustration of optical phenomenn, by Lord Rayleigh.* The present paper takes up the same problem in a more general form; thus in $\S 5$ the obstacle is supposed to be rigid and fixed; in $\$ 6$ it is rigid but movable; we next consider the case of a rigid shell containing a vibratory mechanism ( $\$ 7$ ) or a gyrostat $(\S 8)$; in $\S .9$ the effect of a spherical cavity in the medium is examined; finally, in $\S 10$, we liave the case of an elastic sphere whose density and rigidity may differ to any extent from those of the surrounding medium.

The investigation was undertaken origiually with a view of obtaining a mechanical illustration of the selective absorption of light by a gas, on the hypothesis that this (apparent) absorption is due to a coincidence between the period of the incident waves and that of a free mode of vibration of the molecules. It was found, however, that in this field, as well as in others, the electromagnetic theory furuishes in some respects a much more complete analogy with optical conditions. It has also the advantage of greater mathematical simplicity. For these reasons the calculations of this paper were for a time suspended; they are now completed and published in the hope that they may still present some features of dynamical interest.

The point above referred to, having been in the meantime discussed at length in the papers cited below, is here treated more briefly in $\$ 11$. It appears that the law of dissipation of radiant energy by scattering (when this is a maximum) has the same form as in the acoustical analogy considered in a former paper, $t$ viz., we tind that the ratio $(I)$ which the energy dissipated outwards per.

- Phil. Mag., June, 1871 ; Scientifir Papcrs. Vol. 1., p. 104.

+ "A Problem in Resonance, illustrative of the Mechanical Theory of Selective Absorption of Light by a Gas," Proc. Lond. Arath. Soc., Vol. Xxxir., p. 11. 
unit time from the sphere bear's to the energy-flux in the incident waves is given by

$$
I=\frac{2 n+1}{2 \pi} \lambda^{2}
$$

where $\lambda$ is the wave-length, and $n$ is the index of the splierical harmonic belonging to the particular type of free vibration in question. It is remarkable that this ratio is independent of the size or constitution of the spherical obstacle, and involves only the wavelength. The saine law (as might be expected from the nature of the analysis) is met with in the corresponding electro-magnetic problem,** and appears to have a very wide range of generality.

The notation for the various solutions of the differential equation

$$
\frac{d^{2} F}{d \zeta^{2}}+\frac{2(n+1)}{\zeta} \frac{d F}{d \zeta}+F=0
$$

which are required in investigations such as the present can hardly be said to be definitely fixed. In the anthor's Hydrodynamics, $t$ it, has been proposed to write

$$
\begin{aligned}
& \psi_{n}(\zeta)=(-)^{n}\left(\frac{d}{\zeta d \zeta}\right)^{n} \sin \zeta \\
& =\frac{1}{1.3 \ldots(2 n+1)}\left\{1-\frac{\zeta^{2}}{2(2 n+3)}+\frac{\zeta^{4}}{2.4(2 n+3)(2 n+5)}-\ldots\right\} \text {, } \\
& \Psi_{n}(\zeta)=(-)^{n}(\underset{\zeta}{\dot{\zeta} \dot{d} \xi}) \frac{\cos \zeta}{\zeta} \\
& =\frac{1.3 \ldots(2 n-1)}{\zeta^{2 n+1}}\left\{1-\frac{\zeta^{2}}{2(1-2 n)}+\frac{\zeta^{4}}{2.4(1-2 n)(3-2 n)}-\ldots\right\} \text {, } \\
& f_{n}(\zeta)=(-)^{n}\left(\frac{d}{\zeta d \zeta}\right)^{n} \frac{e^{-\ell \zeta}}{\zeta}=\Psi_{n}(\zeta)-i \psi_{n}(\zeta)
\end{aligned}
$$

these notations will be adhered to in the present paper. The above

- Camb. Trans., Vol. xvrrr., p. 348. [See also L. Lorenz, CEutres Scientifiqtes, Copenhrgen, 1898 , t. 1., p. 499 , where tho formula (1) is arrived at from a somewhat different basis. The investigation was published (in Danish) in 1890: the writer regrets that he has only recently become acquainted with it. - Sept., 1900.]

$+\$ \$ 267,309$. 
122 Prof. Horace Lamb on the Impact of Waves on a [Mar. 8 , functions all satisfy equations of the types

$$
\begin{gathered}
\psi_{n}^{\prime}(\zeta)=-\zeta \psi_{n+1}(\zeta), \\
\zeta \psi_{n}^{\prime}(\zeta)+(2 n+1) \psi_{n}(\zeta)=\psi_{n-1}(\zeta),
\end{gathered}
$$

which are frequently required in reductions. We shall also have occasion to appeal to the formula

$$
x \phi_{n}=\frac{r^{2}}{2 n+1}\left(\frac{1 \cdot \phi_{n}}{d x}-r^{3 n+1} \frac{d}{d x} \frac{\phi_{n}}{r^{2 n+i}}\right)
$$

und its companions, where $\phi_{n}$ is a solid harmonic of (positive or negative) degree $n$.

\section{General Equations.}

1. The component displacements $(u, v, w)$ at any point of an incompressible isotropic medium satisfy the equations

$$
\begin{gathered}
\frac{d^{9} u}{d t^{8}}=c^{8} \nabla^{2} u+\frac{1}{\rho} \frac{d p}{d x}, \frac{d^{2} v}{d t^{3}}=c^{2} \nabla^{\ddagger} v+\frac{1}{\rho} \frac{d p}{d y}, \frac{d^{2} w}{d t^{9}}=c^{2} \nabla^{2} v+\frac{1}{\rho} \frac{d p}{d z}, \\
\frac{d u}{d x}+\frac{d v}{d y}+\frac{d w}{d z}=0,
\end{gathered}
$$

where $c$ is the wave-velocity, connected with the rigidity $(\mu)$ and the density $(\rho)$ by the relation

$$
c^{2}=\mu / \rho,
$$

and $p$ denotes the mean normal traction about the point $(x, y, z)$. From these we deduce

$$
\nabla^{2} p=0
$$

In the case of simple-harmonic motion we assume a time-factor $e^{\text {rkrt }}$, so that $2 \pi / k$ denotes the wave-length of plane waves of the same frequency. The equations (9) then take the forms

$$
\left(\Gamma^{2}+k^{2}\right) u=-\frac{1}{\mu} \frac{d p}{d x},\left(\nabla^{2}+k^{2}\right) v=-\frac{1}{\mu} \frac{d p}{d y},\left(\nabla^{2}+k^{2}\right) w=-\frac{1}{\mu} \frac{d p}{d z} .
$$

In the problems which we have in view the medium is limited internally by a spherical surface (of radius $a$ ) described about the origin, and the disturbance may be regarded as made up of two parts, viz., the extraneous disturbance due to given sources at a distance, and the waves scattered outwards by the sphere. 
1900.] Spherical Obstacle in an Elastic Medium.

If the medium were uninterrupted, the displacements due to the extraneous disturbance would be finite at the origin. Hence for the incident wave-system we may write

where

$$
\left.\begin{array}{c}
p=-\mu k^{2} \Sigma r^{n} S_{n} \\
u=\Sigma u_{n}, \quad v=\Sigma v_{n}, \quad w=\Sigma w_{n}
\end{array}\right\}
$$

$$
\begin{array}{r}
u_{n}=\frac{d}{d x} r^{n} S_{n}+(n+1) \psi_{n-1}(k r) \frac{d}{d x} r^{n} T_{n}-u k^{2} r^{2 n+3} \psi_{n+1}(k r) \frac{d}{d x} \frac{T_{n}}{r^{n+1}} \\
+\psi_{n}(k r)\left(y \frac{d}{d z}-z \frac{d}{d y}\right) r^{n} U_{n} \\
v_{n}=\frac{d}{d y} r^{n} S_{n}+(n+1) \psi_{n-1}(k r) \frac{d}{d y} r^{n} T_{n}-n k^{2} r^{2 n+3} \psi_{n+1}(k r) \frac{d}{d y} \frac{T_{n}}{r^{n+1}} \\
+\psi_{n}(k r)\left(z \frac{d}{d x}-x \frac{d}{d z}\right) r^{n} U_{n} \\
\begin{array}{r}
w_{n}=\frac{d}{d z} r^{n} S_{n}+(n+1) \psi_{n-1}(k r) \frac{d}{d z} r^{n} T_{n}-n k^{2} r^{2 n+3} \psi_{n+1}(k r) \frac{d}{d z} \frac{T_{n}}{r^{n+1}} \\
+\psi_{n}(k r)\left(x \frac{d}{d y}-y \frac{d}{d x}\right) r^{n} U_{n}
\end{array}
\end{array}
$$

The function $\psi_{n}(k r)$ is defined by (3), and $S_{n}, T_{n}, U_{n}$ are spherical surface-harmonics of order $n$. The sign $\Sigma$ indicates summation with respect to $n$, which may have the values $1,2,3, \ldots$. These formulø make

$$
\begin{gathered}
x u+y v+z w=\Sigma\left\{n r^{n} S_{n}+n(n+1)(2 n+1) \psi_{n}(k r) r^{n} T_{n}\right\}, \quad \text { (16) } \\
x\left(\frac{d w}{d y}-\frac{d v}{d z}\right)+y\left(\frac{d u}{d z}-\frac{d w}{d x}\right)+z\left(\frac{d v}{d x}-\frac{d u}{d y}\right)=-\Sigma n(n+1) \psi_{n}(k r) r^{n} U_{n}
\end{gathered}
$$

When the extraneous disturbance consists of a train of plane waves, the harmonics $S_{n}, T_{n}, U_{n}$ are determined as follows. Assuming that the direction of propagation is that of $x$-negative, and that the vibration is parallel to $y$, we may write, symbolically,

$$
u=0, \quad v=e^{i k x}, \quad v=0,
$$

the time-factor $e^{\text {iket }}$ being omitted, here and elsewhere, for shortness. If this be resolved into the sum of a series of disturbances of the 
124. Prof. Horace Lamb on the Impact of Waves on a [Mar. 8, type (15), we must have, by (16) and (17),

$$
\begin{gathered}
\Sigma\left\{n r^{n} S_{n}+n(n+1)(2 n+1) \psi_{n}(k r) r^{n} T_{n}\right\}=y e^{i k x}, \\
\Sigma n(n+1) \psi_{n}(k r) r^{n} U_{n}=-i k z e^{i k x} .
\end{gathered}
$$

Now, if we put

$$
.=r \cos \theta, \quad y=r \sin \theta \cos \omega, \quad z=r \sin \theta \sin \omega,
$$

we have iky $e^{i k x}=\Sigma(2 n+1)(i k r)^{n} \psi_{n}(k r) \sin \theta \cos \omega P_{n}^{\prime}(\cos \theta)$,

where $P_{n}(\cos \theta)$ is the ordinary zonal harmonic. We infer, by comparison with (19), that $S_{n}=0$, and

$$
T_{n}^{\prime}=\frac{(i k)^{n-1}}{n(n+1)} \sin \theta \cos \omega P_{n}^{\prime}(\cos \theta) .
$$

Similarly, we find

$$
U_{n}=-\underset{n i(n+1)}{2 n+l}(i k)^{n} \sin \theta \sin \omega P_{n}^{\prime}(\cos \theta) .
$$

In particular,

$$
T_{1}^{\prime}=\frac{1}{2} \sin \theta \cos \omega=\frac{1}{2} \frac{y}{r}, \quad U_{1}=-\frac{3}{2} i k \sin \theta \sin \omega=-\frac{3}{2} i k_{,}^{2},
$$

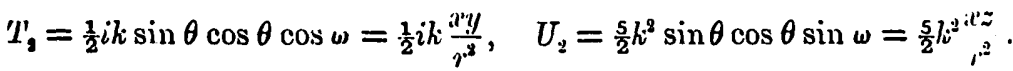

The component tiactions $p_{r x}, p_{r y}, p_{r=}$ on a spherical surface of radius $r$ described about the origin are given by

$$
\left.\begin{array}{l}
r p_{r x}=x p+\mu\left(r \cdot \frac{d}{d r}-1\right) u+\mu \frac{l}{d x}(x u+y v+z w) \\
r p_{r v}=y p+\mu\left(r \frac{d}{d r}-1\right) v+\mu \frac{d}{d y}(x u+y v+z z v) \\
r p_{r z}=z p+\mu\left(\frac{d}{r}-1\right) w+\mu \frac{d}{d z}(x u+y v+z w)
\end{array}\right\}
$$

The parts of $p_{x x}, p_{y,}, p_{r=}$ which arise from $\left(u_{n}, v_{n}, w_{n}\right)$ may be conveniently denoted by $F_{n}, G_{n}, H_{n}$. Their values are written lown here for reference, although they are not really required until $\S 6$. 
We have

$$
\begin{aligned}
& \left.v F_{n}{ }^{\prime} \mu=A_{n}(k v) \frac{d}{d x} r^{n} S_{n}+B_{n}(k r) r^{2 n+1} \frac{d}{d x} \frac{S_{n}}{r^{n+i}}+C_{n}(k v){ }_{d i}^{\prime} r^{n n} T_{n}\right) \\
& +D_{n}(k v) r^{2 n+1} \frac{d}{d x} \frac{T_{n}}{r^{n+1}}+E_{n}(k v)\left(y \frac{d}{d z}-z \frac{d}{d y}\right) r^{n} U_{u} \\
& v G_{n} / \mu=A_{n}(k r) \frac{d}{d y} r^{n} S_{n}+B_{n}(k r) r^{2 n+1} \frac{d}{d y} \frac{S_{n}}{\gamma^{n+1}}+O_{n}(k r) \frac{d}{d y} r^{n} T_{n} \\
& +D_{n}(k r) r^{2 n+1} \frac{d}{d y} \cdot \frac{T_{n}^{\prime}}{r^{n+1}}+E_{u}(k r)\left(z-\frac{d}{d x}-x \frac{d}{d z}\right) r^{n} U_{n} \\
& r H_{n} / \mu=A_{n}(k r) \frac{d}{d z} \cdot{ }^{n} S_{n}+B_{n}(k r) r^{2 n+1} \frac{d}{d z} \frac{S_{n}}{r^{n+1}}+C_{n}(k v) \frac{d}{d z} \cdot r^{n} T_{n} \\
& \left.+D_{n}(k r) r^{m+1} \frac{d}{d z} T_{r^{n+i}}+E_{n}(k v)\left(x \frac{d}{d y}-y \frac{d}{d x}\right) v^{n} U_{n}\right)
\end{aligned}
$$

$$
\begin{aligned}
& \text { where } \left.\quad \begin{array}{rl}
\quad A_{n}(k v) & =-\frac{k^{2} v^{2}}{2 n+1}+2(n-1) \\
B_{n}(k v) & =\frac{h^{2} v^{9}}{2 n+1}
\end{array}\right\}, \\
& \left.\begin{array}{l}
G_{n}(k r)=(n+1)\left\{k r \psi_{n-1}^{\prime}(k r)+2(n-1) \psi_{n-1}(k r)\right\} \\
D_{n}(k r)=-n k^{2} r^{3}\left\{k r \psi_{n+1}^{\prime}(k r)-\psi_{n+1}(k r)\right\}
\end{array}\right\}, \\
& E_{n}(k r)=k r \psi_{n}^{\prime}(k r)+(n-1) \psi_{n}(k r) . *
\end{aligned}
$$

In the case of plane waves the terms in $S_{n}$, as we have seen, are alisent.

\section{Divergent Wares.}

2. We have next to consider the expressions for the divergent waves. For these we have

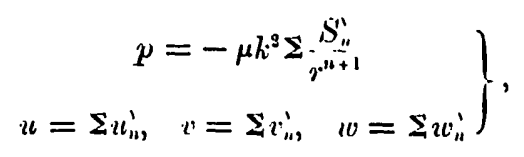

\footnotetext{
* The steps of the calculation are indicated in Hydrodynamics, $\$ 306$.
} 
where

$$
\begin{aligned}
& u_{n}^{\prime}=\frac{d}{d x} \frac{S_{n}^{\prime}}{r^{n+1}}+(n+1) f_{n-1}(k v) \frac{d}{d x} r^{n} T_{n}^{\prime}-n k^{3} r^{2 n+3} f_{n+1}(k r) \frac{d}{d x} \frac{T_{n}^{\prime}}{r^{n+1}} \\
& +f_{n}(k r)\left(y \frac{d}{d z}-z \frac{d}{d y}\right) r^{n} U_{n}^{\prime} \\
& v_{n}^{\prime}=\frac{d}{d y} \frac{S_{n}^{\prime}}{r^{n+1}}+(n+1) f_{n-1}(k r) \frac{d}{d y} r^{n} T_{n}^{\prime}-n k^{2} \eta^{2 n+3} f_{n+1}(k v) \frac{d}{d y} \frac{T_{n}^{\prime}}{r^{n+1}} \\
& \left.+f_{n}(k v)\left(z \frac{d}{d x}-x \frac{d}{d z}\right) r^{n} U_{n}^{\prime}\right\}^{\prime} \\
& w_{n}^{\prime}=\frac{d}{d z} \underset{\gamma^{n+1}}{\stackrel{S_{n}^{\prime}}{\prime}}+(n+1) f_{n-1}(k r) \frac{d}{d z} r^{n} T_{n}^{\prime}-n k^{2} \eta^{2 n+3} f_{n+1}(k r) \frac{d}{d z} \frac{T_{n}^{\prime}}{\gamma^{n+1}} \\
& +f_{n}(k v)\left(a^{2} \frac{d}{d y}-y \frac{d}{d x}\right) r^{n} U_{n}^{\prime}
\end{aligned}
$$

$f_{n}\left(k v^{\circ}\right)$ being the function defined by (5). The surface-harmonics $S_{n}^{\prime}, T_{n}^{\prime}, U_{n}^{\prime}$ are to be found in terms of $S_{n}, T_{n}, U_{n}$ by means of the conditions which hold at the surface $r=a$. The admissible values of $n$ are $1,2,3, \ldots$, as before, the case $n=0$ being excluded, since the volume enclosed by the spherical boundary will be assumed to be constant. These formulæ make

$$
\begin{aligned}
& x u+y v+z v=\Sigma\left\{-(n+1) \frac{S_{n}^{\prime}}{r^{n+1}}+n(n+1)(2 n+1) f_{n}(k r) r^{n} T_{n}^{\prime}\right\},(34) \\
& x\left(\frac{d w}{d y}-\frac{d v}{d z}\right)+y\left(\frac{d u}{d z}-\frac{d w}{d x}\right)+z\left(\frac{d v}{d x}-\frac{d u}{d y}\right)=-\Sigma n(n+1) f_{n}(k r) I_{n}^{\prime \prime} .
\end{aligned}
$$

Again, if we denote the parts of $p_{r x}, p_{r y}, p_{r z}$ which are due to $\left(u_{n}^{\prime}, v_{n}^{\prime}, w_{n}^{\prime}\right)$ by $F_{n}^{\prime}, G_{n}^{\prime}, H_{n}^{\prime}$, we find

$$
\begin{aligned}
& \left.r \cdot F_{n}^{\prime} / \mu=A_{n}^{\prime}(k \cdot r) \frac{1}{r^{2 n+1}} \frac{d}{d x} r^{\prime \prime} S_{n}^{\prime}+B_{n}^{\prime}(k r) \frac{d}{d x} \frac{S_{n}^{\prime}}{r^{n+1}}+C_{n}^{\prime}(k r) \frac{d}{d x} r^{\prime \prime} T_{n}^{\prime \prime}\right) \\
& +D_{n}^{\prime}(k r) r^{2 n+1} \frac{d}{d x} \frac{T_{n}^{\prime}}{r^{n+1}}+E_{n}^{\prime}(k r)\left(y \frac{d}{d z}-z \frac{d}{d y}\right) r^{n} U_{n} \\
& r G_{n}^{\prime} / \mu=A_{n}^{\prime}(k r) \frac{1}{\gamma^{\prime 2 n+1}} \underset{d y}{d}{ }^{\prime n} S_{n}^{\prime}+B_{n}^{\prime}(k r) \frac{d}{d y} \frac{S_{n}^{\prime}}{r^{n+1}}+C_{n}^{\prime}(k r) \frac{d}{d y} r^{n} T_{n}^{\prime} \\
& +D_{n}^{\prime}(k r) r^{2 n+1} \frac{d}{d y} \frac{T_{n}^{\prime}}{r^{n+1}}+E_{n}^{\prime}(k r)\left(z \frac{d}{d x}-x \frac{d}{d z}\right) r^{n} U_{n}^{\prime} \\
& r H_{n}^{\prime} / \mu=A_{n}^{\prime}(k r) \underset{\gamma^{2 n+i}}{1} \frac{d}{d z} r^{n} S_{n}^{\prime}+B_{n}^{\prime}(k v) \cdot \frac{d}{d z} \frac{S_{n}^{\prime}}{\gamma^{n+1}}+C_{n}^{\prime}(k v) \frac{d}{d z} r^{n} T_{n}^{\prime} \\
& \left.+D_{n}^{\prime}(k r) r^{2 n+1} \frac{d}{d z} \frac{T_{n}^{\prime}}{r^{n+1}}+E_{n}^{\prime}(k r)\left(x \frac{d}{d y}-y \frac{d}{d i c}\right) r^{n} U_{n}^{\prime}\right)
\end{aligned}
$$




$$
\begin{aligned}
& \text { where } \left.\begin{array}{rl}
A_{n}^{\prime}(k r) & =-\frac{k^{2} r^{2}}{2 n+1} \\
& B_{n}^{\prime}(k r)=\frac{k^{2} r^{2}}{2 n+1}-2(n+2)
\end{array}\right\}, \\
& \left.\begin{array}{l}
C_{n}^{\prime}(k r)=(n+1)\left\{k v f_{n-1}^{\prime}(k v)+2(n-1) f_{n-1}(k r)\right\} \\
D_{n}^{\prime}(k r)=-n k^{2} r^{2}\left\{k r f_{n+1}^{\prime}(k v)-f_{n+1}(k v)\right\}
\end{array}\right\}, \\
& \left.E_{n}^{\prime}(k r)=k r f_{n}^{\prime}(k r)+(n-1) f_{n}(k r)\right\} \text {. }
\end{aligned}
$$

3. We have to examine the properties of the scattered waves at a great distance from the origin. When $k r$ is large, we have

$$
f_{u}(k r)=\frac{i^{n}}{(k r)^{n+1}} e^{-i k r}
$$

upproximately, and therefore, keeping only the most important terms,

$$
\begin{aligned}
& u_{n}^{\prime}=(2 n+1) \frac{i^{n-1}}{(k r)^{n}} e^{-i k r}\left(\frac{d}{d x} r^{n} T_{n}^{\prime}-n k r^{n-2} T_{n}^{\prime}\right) . \\
& +\underset{(k r)^{n+1}}{i^{n}} e^{-i k r}\left(y \stackrel{d}{d z}-z \frac{d}{d y}\right) r^{n} U_{n}^{\prime} \\
& v_{n}^{\prime}=(2 n+1) \frac{i^{n-1}}{(l i r)^{n}} e^{-\imath k r}\left(\frac{d}{d y} v^{n} T_{n}^{\prime}-n y r^{n-2} T_{n}^{\prime}\right) \\
& +\frac{i^{n}}{(k v)^{n+1}} e^{-i k r}\left(z \frac{d}{d x}-x \cdot \frac{d}{d z}\right) r^{\prime \prime} U_{n}^{\prime} \\
& w_{n}^{\prime}=(2 n+1) \frac{i^{n-1}}{(k r)^{n}} e^{-i k r}\left(\frac{d l}{d z}, v^{n} I_{n}^{\prime}-\imath z r^{n-2} I_{n}^{\prime}\right) \\
& \left.+\frac{i^{n}}{(k r)^{n+i}} e^{-i k r}\left(a \frac{l}{d y}-\frac{d l}{y}\right) r^{n} U_{n}^{\prime}\right)
\end{aligned}
$$

where a slight trausformation has been effected by means of the formula (8). We notice that $u_{n}^{\prime}, v_{n}^{\prime}$, wo are ultimately of the order. $1 / r$, whilst the radial displacenient $\left(x u_{n}^{\prime}+y v_{n}^{\prime}+\approx w v_{n}^{\prime}\right) / r$ is zero to the present degree of approximation. It is really of the order $1 / r^{2}$, as is easily seen from (34).

The geometrical interpretation of the two types of terms which appear in (41) is very simple. The ter'ms in $I_{n}^{\prime \prime}$ express a state of motion in which the particles on a sphere of large radius $r$ are 


\section{Prof. Horace Lamb on the Impact of Waves on a [Mar. 8.}

vibrating in directions orthogonal to the contour-lines of the harmonio $T_{n}^{\prime}$, with an amplitude inversely proportional to the distance between consecutive contours, if we imagine these to be drawn for equal infinitesimal increments of ' $T_{n}^{\prime}$. The terms in $U_{n}^{\prime}$, on the other hand, express a vibration along the contour lines of $U_{n}^{\prime}$, with a similar law of amplitude.*

For example, if (in accordance with a later notation) we write

$$
r T_{1}^{\prime}=\frac{1}{2} B_{1}^{\prime} y, \quad r \cdot U_{1}^{\prime}=0,
$$

we have $\quad\left(u i_{1}^{\prime}, v_{1}^{\prime}, w_{1}^{\prime}\right)=\frac{3 B_{1}^{\prime}}{2 k}\left(-\frac{x y}{r^{3}}, \frac{x^{2}+z^{2}}{r^{3}},-\frac{y z}{r^{2}}\right) \frac{e^{-i k r}}{r}$.

The particles on a sphere of large radius $r$ are here vibrating in the directions of the meridians in which the sphere is cut by planes through the axis of $y$, with an amplitude proportional to the distance from this $\Omega \times$ is.

On the other hand, if

$$
r^{\prime} L_{1}^{\prime}=0, \quad r U_{1}^{\prime}=-\frac{3}{2} i k O_{1}^{\prime} z
$$

we find

$$
\left(u_{1}, v_{1}^{\prime}, u_{1}^{\prime}\right)=\frac{3 C_{1}^{\prime}}{2 k}\left(\frac{y}{r},-\frac{x}{r}, 0\right) \frac{e^{-i k r}}{r} .
$$

'I.'he lines of motion are circles about the axis of $z$, and the amplitude, for points at the same distance $r$ from the origin, varies as the distunce from this axis.

$$
\text { Again, if } \quad r^{2} \cdot C_{2}^{\prime}=\frac{1}{2} i k b_{i}^{\prime} x y, \quad U_{2}^{\prime}=0,
$$

we find

$$
\left(u \vdots, v_{i}, w_{: z}^{\prime}\right)=-\frac{5 B}{2 k}\left(\begin{array}{c}
y\left(y^{2}+z^{2}-x^{3}\right), n\left(z^{3}+x^{3}-y^{3}\right) \\
r^{3}
\end{array}, \frac{2 x y z}{r^{3}}\right) \frac{e^{-i k r}}{r} .
$$

'The lines of motion on a sphere of radius $r$ are the orthogonal trajectories of the curves in which the sphere is cut by the hyperbolic cylinders $a y=$ const. It is easily proved that these trajectories are the intersections of the sphere with the cones $z^{3}=\lambda\left(x^{2}-y^{2}\right)$, where $\lambda$ is a variable parmeter. They all pass through the four points

$$
\left( \pm \frac{r}{\sqrt{2} \underline{2}}, \pm \frac{r}{\sqrt{ } 2}, 0\right)
$$

'The values of $F_{n}^{\prime}, G_{n}^{\prime}, H_{n}^{\prime}$ at a great distanco from the origin are

* Cf. Mroc. Lomd. Math. Sor., Vol. xiII., p. 194. 
most easily obtained from (27) and (41). Having regard to the order. of magnitude of $u_{n}^{\prime}, v_{n}^{\prime}, w_{n}^{\prime}$ and $\left(x u_{n}^{\prime}+y v_{n}^{\prime}+z w_{n}^{\prime}\right) / r$, we readily find

$$
F_{n}^{\prime}=-i k \mu u_{n}^{\prime}, \quad G_{n}^{\prime}=-i k \mu v_{n}^{\prime}, \quad H_{n}^{\prime}=-i k \mu w_{n}^{\prime} .
$$

It appears that the radial stress is relatively very small, as might have been anticipated from the fact that the waves tend to become ultimately plane.

\section{Propayation of Energy Outuards.}

4. 'The formulæ (41) and (48) enable us to calculate a very useful expression for the rate at which energy is carried off by the system of scattered waves represented by (32), (33). If, after restoring the time-factor, we take only the real parts of our expressions, the rate in question is equal to the menn value (with respect to the time) of the integral

$$
-\iint\left(p_{r x} \frac{d n}{d t}+p_{r y} \frac{d r}{d t}+p_{r z} \frac{d \imath v}{d t}\right) d s
$$

taken over the surface of a sphere, of any radius $r$, clescribed about the origin. For simplicity of calculation we may suppose $r$ to be infinitely great.

It appers on examination that, if $n$ and $n$ be distinct,

$$
\iint\left(F_{m}^{\prime} \frac{d u_{n}^{\prime}}{d t}+G_{m}^{\prime} \frac{d v_{n}^{\prime}}{d t}+H_{m}^{\prime} \frac{d w_{n}^{\prime}}{d t}\right) d S=0
$$

in virtue of the conjugate property of spherical harmonics of different order's, so that the expression (49) reduces to the form

$$
-\Sigma \iint\left(F_{n}^{\prime} \frac{d u_{n}^{\prime}}{d t}+G_{n}^{\prime} \frac{d v_{n}^{\prime}}{d t}+H_{n}^{\prime} \frac{d u w_{n}^{\prime}}{d t}\right) d s
$$

Moreover, it is easily seen that the terms in this expression which involve combinations of $T_{n}^{\prime}$ with $U_{n}^{\prime}$ will disappear: *

Let us now write in (41)

$$
r^{n} T_{n}^{\prime}=\Phi_{n}+i \phi_{: 1}
$$

Taking real parts, we have, so fin as the terms in ' $T_{n}^{\prime}$ are concerned,

$$
\begin{aligned}
& d u_{n}^{\prime}=k c \underset{(k r)^{n}}{2 n+1}\left\{\left(\frac{d \Phi_{n}}{d a}-n_{r}^{n}{ }_{r}^{n} \Phi_{n}\right) \cos k(c t-r+\epsilon)\right. \\
& \left.-\left(\frac{d \phi_{u}}{d_{l:}}-u \frac{a}{r^{2}} \phi_{u}\right) \sin k(c t-i+\epsilon)\right\},
\end{aligned}
$$

YOL. XXXII. - NO. 718.

- See Camb. Trams., Val. xvin., p. 357. 
130 Prof. Horace Lamb on the Impact of Waves on a [Mar. 8, where $\epsilon$ may be 0 or $\pi$ ol $\pm \frac{1}{2} \pi$. Also

$$
-F_{u}^{\prime \prime}=\mu k \frac{2 n+1}{(k r)^{n}}\{\ldots\},
$$

where the terms in \{\} are the same as in (53). Hence, so far as it depends on $T_{n}^{\prime \prime}$, the mean value of the expression (51) will be

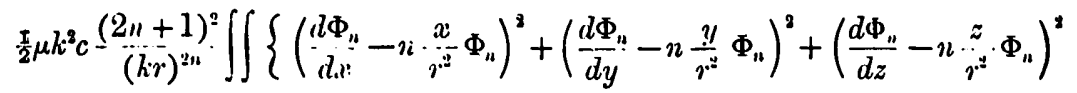

$$
\begin{aligned}
& \left.+\left(\frac{d \phi_{n}}{d x}-n \frac{x}{r^{3}} \phi_{n}\right)^{2}+\left(\frac{d \phi_{n}}{d y}-n \frac{y}{r^{2}} \cdot \phi_{n}\right)^{2}+\left(\frac{d \phi_{n}}{d z}-n \frac{z}{r^{3}} \phi_{n}\right)^{3}\right\} d s \\
& =\frac{r}{2} \mu k^{2} c \frac{(2 n+1)^{2}}{(k r)^{2 n}} \iint\left\{\left(\frac{d \Phi_{n}}{d x}\right)^{2}+\left(\frac{d \Phi_{n}}{d y}\right)^{2}+\left(\frac{d \Phi_{n}}{d z}\right)^{2}-\frac{n^{3}}{\gamma^{2}} \Phi_{n}^{2}\right. \\
& \left.+\left(\frac{d \phi_{n}}{d x}\right)^{2}+\left(\frac{d \phi_{n}}{d y}\right)^{2}+\left(\frac{d \phi_{n}}{d z}\right)^{3}-\frac{n^{2}}{r^{2}} \phi_{n}^{2}\right\} d S .
\end{aligned}
$$

In the same way, if

$$
r^{\prime \prime} U_{n}^{\prime}=\mathbf{X}_{n}+i \chi_{n},
$$

we find that the mean value of (51), so far as it depends on $U_{m}^{\prime}$, will be

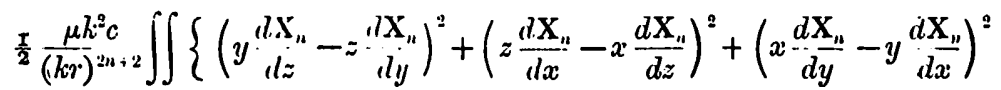

$$
\begin{aligned}
& \left.+\left(y \frac{d X_{n}}{d z}-z^{\prime \prime X_{n}} d^{2}\right)^{2}+\left(z \frac{d \underline{X_{n}}}{d x}-x \frac{d X_{n}}{d z}\right)^{2}+\left(x: \frac{d X_{n}}{d y}-y \frac{d X_{n}}{d x}\right)^{2}\right\} d S \\
& =\frac{x}{2} \frac{\mu c}{(k r)^{2 n}} \iint\left\{\left(\begin{array}{c}
d X_{n} \\
d i x
\end{array}\right)^{2}+\left(\begin{array}{c}
d X_{n} \\
d y
\end{array}\right)^{2}+\left(\frac{d X_{n}}{d z}\right)^{2}-\frac{n^{2}}{r^{2}} \cdot \mathbf{X}_{n}^{2}\right. \\
& \left.+\left(\begin{array}{c}
d x_{n} \\
d x
\end{array}\right)^{2}+\left(\begin{array}{c}
d x_{n} \\
d l_{y}
\end{array}\right)^{2}+\left(\begin{array}{c}
d x_{n} \\
d z
\end{array}\right)^{2}-\frac{n^{2}}{r^{3}} \chi_{n}^{2}\right\} d S .
\end{aligned}
$$

The expressions under the integral signs in (55) and (57) are the sums of the squares of the tangential components of the rectors $\left(d \Phi_{n} / d x, l \Phi_{n} / d l_{y}, d \Phi_{n} / d z\right)$, $d x$., as night have been foreseen from the geometrical interpretation indicated in $\S 8$. Now, turning to polar coordinates, if $\Omega_{n}$ be a surface-har'monic of order $n$, we have

$$
\int_{0}^{2 *} \int_{11}^{*}\left\{\left(\begin{array}{c}
d \Omega_{n}{ }^{2} \\
d \theta
\end{array}\right)^{2}+\left(\begin{array}{c}
d \Omega_{n} \\
\sin \theta d \omega
\end{array}\right)^{2}\right\} \sin \theta d \theta d \omega=u(n+1) \int_{0}^{3 *} \int_{0}^{\infty} \Omega_{n}^{2} \sin \theta d \theta d \omega .
$$


Hence, summing the expressions (55) and (57), we obtain

$$
\frac{1}{2} \mu c \Sigma^{n}(n+1) \int_{0}^{2 \pi} \int_{0}^{\pi}\left\{(2 n+1)^{2} h^{2}\left|T_{n}^{\prime}\right|^{2}+\left|U_{n}^{\prime}\right|^{2}\right\} \sin \theta d \theta d \omega .
$$

In the case of incident plane waves, the formulø (23) and (24) suggest that we slould write

$$
\begin{aligned}
& T_{n}^{\prime}=B_{n}^{\prime} T_{n}=B_{n}^{\prime} \frac{(i k)^{n-1}}{n(n+1)} \sin \theta \cos \omega P_{n}^{\prime}(\cos \theta), \\
& U_{n}^{\prime}=C_{n}^{\prime} U_{n}=-C_{n}^{\prime} \frac{2 n+1}{n(n+1)}(i k)^{n} \sin \theta \sin \omega P_{n}^{\prime}(\cos \theta) .
\end{aligned}
$$

Since $\int_{0}^{2 \pi} \int_{0}^{\pi}\left\{\sin \theta \cos \omega P_{n}^{\prime}(\cos \theta)\right\}^{2} \sin \theta d \theta d \omega=\frac{2 \pi n(n+1)}{2 n+1}$,

the expression (59) now takes the form

$$
\pi \mu c \Sigma(2 n+1)\left\{\left|B_{n}^{\prime}\right|^{2}+\left|C_{n}^{\prime}\right|^{2}\right\} \text {. }
$$

The proper standard of comparison here is the energy propagated per unit time across unit area (of the wave-front) in the primary waves represented by (18). This is equal to $\frac{1}{2} \mu l l^{2} c$. Hence, denoting by $I$ the ratio which the energy dissipated by the scattered waves bears to the energy-flux in the primaly waves, we have

$$
I=\frac{2 \pi}{k^{2}} \Sigma(2 n+1)\left\{\left|B_{n}^{\prime}\right|^{2}+\left|C_{n}^{\prime}\right|^{2}\right\} \text {. }
$$

This has exactly the same form as in the corresponding electromagnetic problem.*

\section{Fined Rigid Obstacle.}

5. We may first suppose that the internal boundary $(r=a)$ is absolutely fixed. The surface-conditions then are

$$
\Sigma\left(u_{n}+u_{n}^{\prime}\right)=0, \quad \Sigma\left(v_{n}+v_{n}^{\prime}\right)=0, \quad \Sigma\left(v_{n}+w_{n}^{\prime}\right)=0 .
$$

If we substitute the values of $u_{n}, v_{n}, v_{n}, u_{n}^{\prime}, v_{n}^{\prime}, w_{n}^{\prime}$ from (15) and (33), we deducet

$$
\begin{aligned}
& S_{n}+(n+1) \psi_{n-1}(k a) T_{n}+(n+1) f_{n-1}(k a) T_{n}^{\prime}=0 \\
&-n k^{2} a^{2} \psi_{n+1}(k a) T_{n}+\frac{S_{n}^{\prime \prime}}{a^{2 n+1}-\imath k^{2} a^{2} f_{n+1}(k a) T_{n}^{\prime}}=0 \\
& \psi_{n}(k a) U_{n}+f_{n}(k a) U_{n}^{\prime}=0 .
\end{aligned}
$$

- Camb. Trans., Vol. xviII., p. 358. [Sce also Lorenz, loc. cit., p. 492.]

+ The proof of these and similar inferences in the sequal is indicated in IIytlrodynamics, $\$ 307$.

$$
\text { К } 2
$$


13. Prof. Horace Lamb on the Impact of Waves on a [Mar. 8, 'l'hese determine the harmonics $s_{n}^{\prime \prime}, T_{n}^{\prime}, U_{n}^{\prime}$ in terms of $S_{n}, T_{n}, U_{n}$, which are regurded as given.

When there is no extraneous disturbance, we have $S_{n}=0, T_{n}=0$, $U_{n}=0$. The free vibrations involving $T_{n}^{\prime}$ have values of $k$ determined by

$$
f_{n-1}(k a)=0
$$

thus, for $n=2$, we find $i k a=-1$. Since the time-factor is $e^{i k e t}$, this gives an "aperiodic" vibration $e^{-c t ; n}$. The free vibrations involving $U_{n}^{\prime}$ have values of $k$ determined by

$$
f_{n}(k a)=0 \text {. }
$$

'The characteristic of all these free vibrations, whether oscillatory or not, is that the "modulus of decay" is comparable with the time a plane wave would take to traverse a distance equal to the radius of the sphere.

In the case of plane incident waves, we lave $S_{n}=0$; and, if we further suppose that the wave-length is large compared with the dimensions of the spherical obstacle, $k a$ will be a small quantity. Hence, keeping only the most important terms, we find

$$
\begin{aligned}
& T_{n}^{\prime}=-\frac{\psi_{n-1}(k a)}{f_{n-1}(k a)} T_{n}=-\frac{(k a)^{2 n-1}}{\{1.3 \ldots(2 n-3)\}^{2}(2 n-1)} T_{n}, \\
& U_{n}=-\frac{\psi_{n}(k a)}{f_{n}(k a)} U_{n}=-\frac{(k a)^{2 n+1}}{\{1.3 \ldots(2 n-1)\}^{2}(2 n+1)} U_{n} .
\end{aligned}
$$

The most important part of the scattered waves is that representerl by $T$. Since, from (71) and (25),

$$
T_{1}^{\prime}=-k a T_{1}^{\prime}=-\frac{1}{2} k a \cdot \frac{y}{r},
$$

the corresponding vibration $\left(u_{i}^{\prime}, v_{i}^{\prime}, w_{i}^{\prime}\right)$ at a distance from the origin is found by making $B_{1}^{1}=-k a$ in (43). This type of vibration is familiar in problems such as we are engaged on, but the amplitude is now much greater than usual, viz., in a ratio of the $\operatorname{order}(k a)^{-2}$. This is accounted for by the abnormal degree of constraint imposed on our medium at a rigid and imnovable boundary. In the corresponding acoustical problem,* where plane waves of sound impinge on a fixed sphere, there is complete freedom of lateral motion at the surface.

- Lord Rayleigh, Theory of Sound, $\$ 334$. 
T'he formula (64) gives for the dissipation-ratio in the present case

approximately.

$$
I=6 \pi a^{2},
$$

\section{Rigid Movable Sphere.}

6. Next suppose that a perfectly rigid but movable sphere is embedded in the medium. The tractions on the surface will reduce to a force $(X, Y, Z)$ and a couple $(L, M, N)$. When the incident wavesystem is that given by (18), the components $Y$ of force and $N$ of couple will (in the absence of gyrostatic influence) be the only ones which do not vanish, and the motion of the sphere will consist of a linear oscillation in the axis of $y$ and an angular oscillation about the axis of $z$. It is convenient, however, in the first instance, to treat the problem more generally. In any case it appears that the only parts of our general formulæ which will contribute anything to the values of $X, Y, Z, L, M, N$ are those for which $n=1$; moreover, that the linear and angular motions of the sphere are independent, the former depending on the harmonics $S_{1}, T_{1}$, and the latter on $U_{1}$. For $n>1$, the deternination of $S_{n}^{\prime}, T_{n}^{\prime}, U_{n}^{\prime}$ in terms of $S_{n}^{\prime}, T_{n}^{\prime}, U_{n}$ will be exactly the same as when the sphere was fixed $(\$ 5)$.

$$
\text { We find } \left.\quad \begin{array}{rl}
X & =\iint\left(F_{1}+F_{1}^{\prime}\right) d S^{\prime} \\
Y & =\iint\left(G_{1}+G_{1}^{\prime}\right) d S \\
Z & =\iint\left(H_{1}+H_{1}^{\prime}\right) d S
\end{array}\right\},
$$

where the integrations extend over the surface $r=a$. Hence, substituting from (28) and (36), we find

$$
\begin{aligned}
& X=-\frac{4}{3} \pi \mu k^{3} a^{3}\left\{\frac{d}{d x} v S_{1}+\frac{1}{a^{3}} \frac{d}{d x} r S_{1}^{\prime}\right\} \\
& +8 \pi \mu k a^{2}\left\{\psi_{0}^{\prime}(k a) \frac{d}{d x} r T_{1}+f_{0}^{\prime}(k a) \frac{d}{d x} r T_{1}^{\prime}\right\}, \\
& l_{l}=-\frac{8}{3} \pi \mu k a^{4}\left\{\psi_{1}^{\prime}(k a) \frac{d}{d x} r U_{1}+f_{1}^{\prime}\left(k a, \frac{d}{d x} \cdot U_{1}^{\prime}\right\},\right.
\end{aligned}
$$


134 Prof. Horace Lamb on the Impact of Waves on a [Mar. 8, with similar equations. We may therefore write

$$
\begin{array}{r}
-\frac{4}{3} \pi \mu k^{2} a^{4}\left(S_{1}+\frac{S_{1}^{\prime}}{a^{5}}\right)+8 \pi \mu k a^{3}\left\{\psi_{0}^{\prime}(k a) l_{1}+f_{11}^{\prime}(k a) I_{1}^{\prime \prime}\right\}=X_{i 2}+Y y+Z z, \\
-\frac{8}{3} \pi \mu k a^{5}\left\{\psi_{1}^{\prime}(k a) U_{1}+f_{1}^{\prime}(k a) U_{1}^{\prime}\right\}=L x+M y+N z
\end{array}
$$

Again, if $(\alpha, \beta, \gamma)$ and $(\xi, \eta, \zeta)$ be the linear and angular displacements of the sphere, the kincmatical relations to be satisfied at the surface $r=a$ are

$$
u_{1}+u_{1}^{\prime}=a+\eta z-\zeta y, \quad v_{1}+v_{1}^{\prime}=\beta+\zeta x-\xi z, \quad u_{1}+w_{1}^{\prime}=\gamma+\xi_{y} y-\eta x .
$$

Hence, by (16), (17), and (34), (35),

$$
\begin{gathered}
a\left(S_{1}-2 \frac{S_{1}^{\prime}}{a^{3}}\right)+6 a\left\{\psi_{1}(k a) T_{1}+f_{1}(k a) T_{1}^{\prime}\right\}=a n+\beta y+\gamma z \\
-a\left\{\psi_{1}(k a) U_{1}+f_{1}(k a) U_{1}^{\prime}\right\}=\xi x+\eta y+\zeta z
\end{gathered}
$$

It follows also from (15) and (33) that we must have

$$
\frac{S_{1}^{\prime}}{a^{3}}-k^{8} a^{2}\left\{\psi_{2}(k a) T_{1}+f_{3}(k a) T_{1}^{\prime}\right\}=0
$$

since the surface-harmonics of order 2 , in the values of $n_{1}+u_{1}^{i}, v_{1}+v_{1}^{i}$, $w_{1}+w_{\text {i must cancel. }}$

We lave, furtleer, the dynamical equations

$$
\begin{array}{ll}
m \frac{d^{2} a}{d t^{2}}=X, & m \frac{\dot{\pi}^{2} \beta}{d t^{3}}=Y, \quad m \frac{d^{3} \gamma}{d t^{z}}=Z, \\
K \frac{d^{9} \xi}{d t^{3}}=L, & K \frac{d^{3} \eta}{d t^{2}}=M, \quad K \frac{d^{2} \zeta}{d t^{3}}=N,
\end{array}
$$

where $m$ denotes the mass of the sphere, and $K$ its moment of inertia. There is an obvious convenience in writing

$$
n \imath=\kappa \cdot \frac{4}{3} \pi \rho a^{3}, \quad K=1 \cdot \frac{4}{3} \pi \rho a^{3} \cdot \frac{2}{5} a^{2} .
$$

Remembering that the time-factor is $e^{i k r t}$, and that $c=\mu / \rho$, the equations (85), (86) give

$$
\begin{aligned}
& X x+Y y+Z z=-\kappa \cdot \frac{4}{3} \pi \mu k^{2} a^{8}(a x+\beta y+\gamma z), \\
& L x+M y+N z=-v \cdot \frac{8}{15} \pi \mu k^{9} a^{5}(\xi x+\eta y+\zeta z) .
\end{aligned}
$$

Hence, from (79) and (82),

$$
(\kappa-1) S_{1}-(2 \kappa+1) \frac{S_{1}^{\prime}}{a^{3}}+6(\kappa-1)\left\{\psi_{1}(k u) T_{1}+f_{1}(k i l) T_{1}^{\prime}\right\}=0 .
$$


Combined with (84) this gives

$$
\begin{aligned}
(\kappa-1) S_{1}+\left\{(2 \kappa+1) \psi_{0}(k a)\right. & \left.-9 \psi_{1}(k a)\right\} T_{1} \\
& +\left\{(2 \kappa+1) f_{0}(k a)-9 f_{1}(k a)\right\} T_{1}^{\prime}=0
\end{aligned}
$$

where a simplification has been made by means of (6) and (7).

Similarly, from (80), (83), and (89), we deduce

$$
\left\{\frac{1}{5} \nu k a \psi_{1}(k a)+\psi_{1}^{\prime}(k a)\right\} U_{1}+\left\{\frac{1}{5} \nu k a f_{1}(k x)+f_{1}^{\prime}(k a)\right\} U_{\mathrm{i}}^{\prime}=0 .
$$

The free rectilinear vibrations which the sphere can execute in the absence of extraneous disturbance are found by putting $S_{1}=0$, $T_{1}=0$ in (91), whence

or

$$
\begin{aligned}
(2 \kappa+1) f_{0}(k a)-9 f_{1}(k a) & =0, \\
(2 \kappa+1)(i k a)^{2}+9(i k a)+9 & =0 .
\end{aligned}
$$

If $\kappa>\frac{5}{8}$, the roots of this quadratic in $i k a$ are complex, with a real negative part, , viz.,

$$
i k a=-\frac{9}{4 \kappa+2} \pm i \frac{3 \sqrt{ }(8 \kappa-5)}{4 \kappa+2}
$$

This indicates a periodic oscillation with a gradually diminishing amplitude. When the ratio $(k)$ which the mass of the sphere bears to that of an equal volume of the medium is a large number, the wave-length corresponding to the period will be large compared with the radius of the sphere, and a considerable number of oscillations will take placc in the interval required for the amplitude to diminish in the ratio $1 / e$. When $x<\frac{5}{8}$, the values of $i k a$ are real and negative, and the vibration is aperiodic.

Again, the free rotational vibrations of the sphere are found by putting $U_{1}=0$ in (92); thus

$$
\begin{gathered}
\frac{1}{5} \nu k a f_{1}(k a)+f_{1}^{\prime}(k a)=0, \\
\nu(i k a)^{3}+(\nu+5)(i k a)^{2}+15 i k a+15=0 .
\end{gathered}
$$

When $v$ is large one root of this cubic in $i k a$ is $-1-\frac{1}{5} \nu$, nearly, corresponding to an aperiodic motion. The others are given by

$$
i k a=-\frac{75}{2 \nu^{2}} \pm i \mathcal{V}\left(\frac{15}{\nu}\right),
$$

approximately; and it is to be observed that the modulus of decay is, relatively to the period, very much longer than in the case of the ectilinear vibrations. 
In the case of the plane incident system (18) we have $S_{1}=0$, and the values of $T_{1}^{\prime}$ and $U_{1}^{\prime}$ in terms of $T_{1}$ and $U_{1}$, respectively, are given by (91) and (92). When $\kappa$ and $\nu$ have only moderate values, simple approximate expressions are easily found for the case where $k a$ is small; thus

$$
T_{1}=\frac{2}{9}(\kappa-1) k^{3} a^{3} T_{1}, \quad U_{1}=\frac{1}{45}(\nu-1) k^{5} a^{5} U_{1},
$$

showing that the angular motion of the sphere is of secondary im. portance. We must remember that $T_{2}^{\prime}$ is now of equal rank with $T_{1}^{\prime}$, being given by (71). The most important parts of the disturbance at a distance are therefore found by putting

$$
B_{1}^{\prime}=\frac{2}{9}(\kappa-1) k^{3} a^{3}, \quad B_{2}^{\prime}=-\frac{1}{3} k^{3} a^{3},
$$

in (43) and (47) respectively. The dissipation-ratio. is

$$
I=\left\{\frac{8}{27}(\kappa-1)^{2}+\frac{40}{81}\right\} \pi a^{2}(k a)^{4},
$$

approximately, showing the usual law of dependence upon wavelength.

The expressions (99) vanish, to our present degree of approximation, when $\kappa=1, v=1$. For $\kappa=0, v=0$ they coincide with the results obtained below in $\S 9$ for the case of a spherical cavity in the medium.

When, ou the other hand, $\kappa$ and $v$ are so large as to be comparable with $(k a)^{-2}$, the above approximation is insufficient. I.t is clear however that, except in the neighbourhood of the critical wavelengths referred to in $\S 11$, the results must tend to coincide with those obtained in $\S 5$ for a fixed sphere, and in particular the dissipation-ratio must tend to the value (74).

\section{Rigid. Shell containing a Vibratory Mechanism.}

7. The investigation of the preceding section may be readily extended in various ways, by imagining that the sphere is hollow and contains a dynamical system capable of storing potential and kinetic energy. Various arrangements of this kind have been suggested by Lord Kelvin. As a sufficient example of a system devoid of gyrostatic influence, we may take the case of a rigid spherical shell containing a central spherical nucleus attached to it by springs.

To calculate first the linear vibration parallel to $y$, such as will be called into play by waves of the type (18), let $m$ denote the mass of the shell, $m_{0}$ that of the nucleus, and let $\beta, \beta_{0}$ be the displacements of 
their centres. If $2 \pi / \sigma_{0}$ be the period of vibration of the nucleus when the shell is held fixed, we have

and

$$
\begin{aligned}
& \frac{d^{2} \beta_{0}}{d t^{2}}=-\sigma_{0}^{2}\left(\beta_{0}-\beta\right) \\
& m \frac{d^{2} \beta}{d t^{2}}+m_{0} \frac{d^{2} \beta_{0}}{d t^{2}}=Y,
\end{aligned}
$$

where $Y$ denotes, as before, the effect of the stresses on the outer. surface. Writing $-k^{2} c^{2}$ for $d^{2} / d t^{2}$, and eliminating $\beta_{0}$, we find

$$
-\frac{(m+m n) \sigma_{0}^{2}-m k^{2} c^{2}}{\sigma_{0}^{2}-k^{2} c^{2}} k^{2} c^{2} i^{2}=Y .
$$

If we put

$$
m=\kappa \cdot \frac{4}{3} \pi \rho a^{3}, \quad m_{0}=\kappa_{0} \cdot \frac{4}{3} \pi \rho a^{3},
$$

it appears that to adapt the various results of $\S 6$ to the present circumstances we have merely to replace $\kappa$ by

$$
\kappa^{\prime}=\begin{gathered}
\left(\kappa+\kappa_{0}\right) \sigma_{0}^{2}-\kappa k^{2} c^{2} \\
\sigma_{0}^{2}-k^{2} c^{2}
\end{gathered}
$$

The limiting form of the result when the inertia of the nucleus is very great is obtained by putting $\kappa_{0}=\infty, \sigma_{0}=0$, whilst $\kappa_{0} \sigma_{0}^{2}$ is finite. The shell then behaves as if (in addition to the force $Y$ ) it were urged to a fixed position by a force varying as the distance.

Similarly, if the springs resist rotation of the nucleus relatively to the containing shell, we have to replace $v$ by

$$
\nu^{\prime}=\frac{\left(\nu+\nu_{n}\right) \sigma_{n}^{2}-\nu k^{2} c^{2}}{\sigma_{0}^{2}-k^{2} c^{2}}
$$

where the constants $\nu, \nu_{j}, \sigma_{0}$ have analogous meanings, as regards rotation, to those adopted for $\kappa, \kappa_{0}, \sigma_{0}$ in the case of translation.

It is evident in each case that the approximations we have made on the assumption that $k a$ is small will require revision when $k a$ is nearly equal to $\sigma_{0} a / c$. The further consideration of such questions is, lowever, deferred to $\S 11$.

\section{Rigid Shell containing a Gyrestat.}

8. To examine the kind of modification that will be introduced into our results by gyrostatic influence, we may imagine that the sphere of $\S 6$ is replaced by a spherical shell containing a gyrostat in rapid rotation. The first part of the investigation proceeds as before, 
138 Prof. Horace Lamb on the Impact of Waves on a [Mur. 8, until we come to the dynamical equations of rotation (86). These now take the forms

$$
\left.\begin{array}{l}
K \frac{d^{2} \xi}{d t^{3}}+R \frac{d \eta}{d t}-Q \frac{d \zeta}{d t}=I \\
K \frac{d^{2} \eta}{d t^{2}}+P \frac{d \zeta}{d t}-R \frac{d \xi}{d t}=M \\
K \frac{d^{2} \zeta}{d t^{3}}+Q \frac{d \xi}{d t}-P \frac{d \eta}{d t}=N
\end{array}\right\} .
$$

The symbols $P, Q, R$ here denote the components of angular momentum of the gyrostat when the containing shell is at rest in its equilibrium position.

Special interest attaches to the two cases where the axis of the gyrostat is parallel and perpendicular, respectively, to the direction of propagation of the plane waves.

In the former case we have $Q=R=0$, and the equations (108) reduce to

$$
K \frac{d^{3} \xi}{d t^{2}}=L, \quad K \frac{d^{2} \eta}{d t^{8}}+P \frac{d \zeta}{d t}=M, \quad K K_{d t^{2} \zeta}^{d^{2}}-P \frac{d \eta}{d t}=N .
$$

The last two are equivalent to

$$
\left.\begin{array}{l}
K \frac{d^{2}}{d t^{2}}(\eta+i \zeta)-i P \frac{d}{d t}(\eta+i \zeta)=M+i N \\
K \frac{d^{2}}{d t^{2}}(\eta-i \zeta)+i P \frac{d}{d t}(\eta-i \zeta)=M-i N
\end{array}\right\} .
$$

The rotational oscillations about the axis of $x$ call for no special treatment. In the remaining types we have $\xi=0, L=0$, and either

with

$$
\left.\begin{array}{r}
\eta=i \zeta, \quad M=i N \\
K \frac{d^{2} \eta}{d t^{2}}-i P \frac{d \eta}{d t}=M
\end{array}\right\},
$$

$o r$

$$
\left.\begin{array}{l}
\eta=-i \zeta, \quad M=-i N \\
K \frac{d^{2} \eta}{d t^{3}}+i P \frac{d \eta}{d t}=M
\end{array}\right\} .
$$

In the case of (111) we may write

$$
r U_{1}=B(y-i z), \quad r U_{1}^{\prime}=B^{\prime}(y-i z) .
$$


Hence, from (83) and (80),

$$
\begin{aligned}
\eta & =-\left\{B \psi_{1}(k a)+B^{\prime} f_{1}(k a)\right\}, \\
M & =-\frac{8}{3} \pi \mu k a^{4}\left\{B \psi_{1}^{\prime}(k a)+B^{\prime} f_{1}^{\prime}(k a)\right\} .
\end{aligned}
$$

If we write $\quad K=\nu \cdot \frac{4}{3} \pi \rho a^{3} \cdot \frac{2}{5} a^{2}, \quad P=K \varpi$,

w being a quantity of the nature of an angular velocity, and if we assume the time-factor $e^{\text {ikct }}$ in (111), we find

$$
\begin{aligned}
& \left\{\frac{1}{5} \nu\left(k a-\frac{\sigma a}{a}\right) \psi_{1}(k a)+\psi_{1}^{\prime}(k a)\right\} B \\
& +\left\{\frac{1}{5} \nu\left(k a-\frac{\varpi a}{c}\right) f_{1}(k a)+f_{1}^{\prime}(k a)\right\} B^{\prime}=0,
\end{aligned}
$$

which is the altered form of (92).

In the free oscillations of the type (111) we have $B=0$, and

$$
\begin{gathered}
\frac{1}{5} \nu\left(k a-\frac{\varpi a}{c}\right) f_{1}(k a)+f_{1}^{\prime}(k a)=0, \\
\text { or } \quad \frac{1}{5} \nu i k a(i k a+1)\left(i k a-i \frac{\varpi a}{c}\right)+(i k a)^{2}+3 i k a+3=0 .
\end{gathered}
$$

If $v$ is a large number, one root of this is $i k a=-1$, nearly; this indicates a rapidly decaying vibration. If, further, $\sigma a / c$ be small compared with $\sqrt{ }(15 / v)$, the remaining roots will be

$$
\begin{gathered}
i k a=i k_{1} a, \quad-i k_{2} a, \\
k_{1} a=\sqrt{ } \frac{15}{\nu}+\frac{1}{2} \frac{\varpi a}{c}, \quad k_{2} a=\sqrt{ } \frac{15}{\nu^{\prime}}-\frac{1}{2} \frac{\varpi a}{c},
\end{gathered}
$$

where

nearly. It may be shown that in the motion corresponding to the positive value of $k$ the axis of the gyrostat has a positive precessional motion about the axis of $x$, whilst the negative root gives a negative precessional motion. The period $\left(2 \pi / k_{1} c\right.$ or $\left.2 \pi / k_{3} c\right)$ is shorter when the direction of the precessional motion agrees with that of the rotation of the gyrostat than when it is opposed to it.

If we substitute from (11:3) in (41), we find that at a distance from the sphere

$$
\left(u_{1}^{\prime}, v_{l}^{\prime}, w_{1}^{\prime}\right)=\frac{i B^{\prime}}{k^{2} r}\left(-\frac{i y+z}{r}, \frac{i x}{r}, \frac{x}{r}\right)\left\{\begin{array}{l}
e^{i k_{1}(c t-r)} \\
e^{-i k_{2}\left(c t-r_{1}\right.}
\end{array} .\right.
$$


1.40 Prof. Horace. Lamb on the Impact of Waves on a [Mar. 8,

At points on the axis of $x$ the vibrations are circular; in the wave corresponding to $k_{1}$ they have the same sense as $\sigma$, and in the wave corresponding to $k_{8}$ they have the sense opposite to that of $\sigma$. At points of the plane $y z$ the vibrations are rectilinear, and parallel to $x$.

The consideration of the system (112) leads to similar conclusions.

To examine the effect of the obstacle on a system of plane waves it is necessary to analyse these into right-handed and left-handed circular vibrations. For a train of right-handed circular vibrations, say

$$
u=0, \quad v=e^{i k x}, \quad w=-i e^{i k x},
$$

the formulø (113) will apply, provided $B=\frac{3}{2} k$. The value of $b^{\prime}$ is then given by (117), and the scattered waves by

$$
\left(u_{1}^{\prime}, v_{1}^{\prime}, w_{1}^{\prime}\right)=\frac{i B^{\prime}}{k^{3} r}\left(-\frac{i y+z}{r}, \frac{i x}{r}, \frac{x}{r}\right) e^{i k(\mathrm{ct}-r)},
$$

which has just been interpreted. The case of a train of left-handed vibrations could be treated in a similar manner, but the result will evidently be equivalent to reversing the sign of $w$ in (117).

To investigate the case where the axis of the gyrostat is perpendicular to the direction of propagation of the plane waves, it will be sufficient to take the case where the vibrations in these are rectilinear, as expressed by (18), and the axis of the gyrostat is paralle] to the direction of vibration. If we put $P=R=0$, the equations (108) reduce to

$$
K \frac{d^{2} \xi}{d t^{2}}-Q \frac{d \zeta}{d t}=L, \quad K \frac{d^{2} \eta}{d t^{2}}=M, \quad K \frac{d^{2} \zeta}{d t^{2}}+Q \frac{d \xi}{d t}=N .
$$

So far as the effect of the plane waves is concerned we may suppose that $\eta=0, M=0$. Also, assuming

$$
r U_{1}=C z, \quad r U_{1}^{\prime}=A^{\prime} x+C^{\prime} z,
$$

we find $\quad \xi=-A^{\prime} f_{1}(k a), \quad \zeta=-\left\{C \psi_{1}(k a)+C^{\prime} f_{1}(k a)\right\}$,

$$
I=-\frac{8}{3} \pi \mu k a^{4} A^{\prime} f_{1}^{\prime}(k a), \quad N=-\frac{8}{3} \pi \mu k a^{4}\left\{C \psi_{1}^{\prime}(k a)+C^{\prime} f_{1}^{\prime}(k a)\right\}
$$

Writing

$$
Q=K_{\omega}
$$


1900.] Spherical Obstacle in an Elastic Medium.

and substituting in (125), we find

$$
\begin{aligned}
& \left\{\frac{1}{5} \nu k a f_{1}(k a)+f_{1}^{\prime}(k a)\right\} A^{\prime}+\frac{1}{5} i \nu \frac{\varpi a}{i} f_{2}(k a) C^{\prime}=-\frac{1}{5} i \nu{ }_{0}^{\varpi a} \psi_{1}(k a) C,(13 C) \\
& -\frac{1}{5} i \nu \frac{w a}{c} f_{1}(k a) A^{\prime}+\left\{\frac{1}{5} \nu k a f_{1}(k a)+f_{1}^{\prime}(k a)\right\} C^{\prime \prime} \\
& \text { whence }=-\left\{\frac{1}{5} \nu k a \psi_{1}(k a)+\psi_{1}^{\prime}(k a)\right\} O,
\end{aligned}
$$

$$
\begin{aligned}
{\left[\left\{\frac{1}{5} \nu k a f_{1}(k a)+f_{1}^{\prime}(k a\}^{2}\right.\right.} & \left.-\left\{\frac{1}{5} \nu \frac{\varpi a}{c} f_{1}(k a)\right\}^{2}\right] \frac{A^{\prime}}{C} \\
& =-i \nu \frac{\varpi a}{c}\left\{\psi_{1}(k a) f_{1}^{\prime}(k a)-f_{1}(k a) \psi_{1}^{\prime}(k a)\right\} \\
& =i \nu \frac{\varpi a}{c} \frac{1}{k^{4} a^{4}}
\end{aligned}
$$

and

$$
\begin{aligned}
& {\left[\left\{\frac{1}{5} \nu k a f_{1}(k a)+f_{1}^{\prime}(k a)\right\}^{2}-\left\{\frac{1}{5} \nu \frac{\varpi a}{c} f_{1}(k a)\right\}^{2}\right] \frac{C^{\prime}}{C}} \\
& =-\left[\frac{1}{5} \nu k a \psi_{1}(k a)+\psi_{1}^{\prime}(k a)\right\}\left\{\frac{1}{5} \nu k a f_{1}(k a)+f_{1}^{\prime}(k a)\right\} \\
& \left.-\nu^{2} \frac{\omega^{2} a^{2}}{c^{3}} \psi_{1}(k a) f_{1}(k a)\right] .
\end{aligned}
$$

If we substitute from (126) in (41), the component displacements at a distance from the origin are given by

$$
\left(u_{i}^{\prime}, v_{1}^{\prime}, w_{i}^{\prime}\right)=\frac{i}{i_{i}^{i} r}\left(\frac{C^{\prime} y}{r}, \frac{A^{\prime} z-C^{\prime} x}{r},-\frac{A^{\prime} y}{r}\right) e^{i k(t a t-r)} .
$$

For sufficiently small values of $\approx$ the value of $O^{\prime}$ will be sensibly the same as in the problem of $\S 6$, whilst $A^{\prime}$ will be relatively small. The chief effect of the terms in $A^{\prime}$ will be to introduce a small vibration parallel to $y$ at points on the axis of $z$, whilst at points on the axis of $y$ the previously rectilinear vibration will become elliptic, the elliptic orbits being very elongated, with their major axes parallel to the axis of $x$.

\section{Spherical Cavity.}

9. By way of contrast with the problem of $\S 5$, we may next consider the case where the inner boundary $r=a$ is free from stress; in other words, there is a spherical carity in the medium. It 
142 Prof. Horace Lamb on the Impact of Waves on a [Mar. 8, appears from (28) and (36) that the surface-conditions

$$
\Sigma\left(F_{n}+F_{n}^{\prime}\right)=0, \quad \Sigma\left(G_{n}+G_{n}^{\prime}\right)=0, \quad \Sigma\left(H_{n}+H_{n}^{\prime}\right)=0
$$

will be satisfied, provided

$$
\begin{gathered}
A_{n}(k a) S_{n}+C_{n}(k a) T_{n}+A_{n}^{\prime}(k a) \frac{S_{n}^{\prime}}{a^{2 n+1}}+C_{n}^{\prime}(k a) T_{n}=0, \\
B_{n}(k a) S_{n}+D_{n}(k a) T_{n}+B_{n}^{\prime}(k a) \frac{S_{n}^{\prime}}{a^{2 n+1}}+D_{n}^{\prime}(k a) T_{n}=0 \\
E_{n}(k a) U_{n}+E_{n}^{\prime}(k a) U_{n}=0 .
\end{gathered}
$$

Leaving aside the "free" vibrations, we have, when $k a$ is small,

$$
\left.\begin{array}{c}
A_{n}(k a)=2(n-1), \quad B_{n}(k a)=\frac{k^{2} a^{9}}{2 n+1}, \\
C_{n}(k a)=\frac{2\left(n^{2}-1\right)}{1.3 \ldots(2 n-1)}, \quad D_{n}(k a)=\frac{n k^{3} a^{3}}{1.3 \ldots(2 n+3)} \\
E_{n}^{\prime}(k a)=\frac{n-1}{1.3 \ldots(2 n+1)}
\end{array}\right\},
$$

approximately. Substituting in (136), (137), (138), we find that when $S_{n}=0$, as in the case of incident plane waves,

$$
\begin{aligned}
& T_{n}^{\prime}=\frac{2\left(n^{2}-1\right)(k a)^{2 n-1}}{\{1.3 \ldots(2 n-3)\}^{8}(2 n-1)\left(2 n^{3}+1\right)} T_{n,} \\
& U_{n}=\frac{(n-1)(k a)^{2 n+1}}{\{1.3 \ldots(2 n-1)\}^{3}(2 n+1)(n+2)} U_{n} .
\end{aligned}
$$


The case $n=1$ is exceptional, and demands a closer approximation. We find

$$
\begin{gathered}
A_{1}(k a)=-\frac{1}{3} k^{4} a^{2}, \quad B_{1}(k a)=\frac{1}{3} k^{3} a^{8}, \\
C_{1}(k a)=-\frac{2}{3} k^{2} a^{2}, \quad D_{1}(k a)=\frac{1}{15} k^{3} a^{3}, \quad E_{1}(k a)=-1_{1}^{1} k^{2} a^{8}, \\
A_{1}(k a)=-\frac{1}{3} k^{3} a^{2}, \quad B_{1}^{1}(k a)=-6, \\
O_{1}(k a)=-\frac{2}{k a}, \quad D_{1}^{1}(k a)=\frac{18}{k^{8} a^{8}}, \quad E_{1}^{\prime}(k a)=-\frac{3}{l^{8} a^{3}},
\end{gathered}
$$

and thence

$$
T_{1}^{\prime}=-\frac{2}{9} k^{3} a^{3} T_{1}, \quad U_{1}^{\prime}=-\frac{1}{45} k^{5} a^{5} U_{1}
$$

Since, from (143), $\quad T_{2}^{\prime}=\frac{2}{9} k^{8} a^{8} T_{2}$,

it appears that the most important terms in the expressions for the scattered wave are those which arise from $T_{1}^{\prime}$ and $T_{2}^{\prime}$. In the case of the wave-system represented by (18), we have

$$
T_{\mathrm{i}}^{\prime}=-\frac{1}{9} h^{8} a^{8} \frac{y}{r}, \quad T_{2}^{n}=\frac{1}{9} i k^{4} a^{8} \frac{x y}{r^{2}}
$$

the terms in question are therefore obtained by writing $B_{1}^{1}=-\frac{2}{9} k^{3} \iota^{3}$, $B_{2}^{1}=\frac{2}{6} k^{8} a^{8}$, in (43) and (47). And from (64) the dissipation-ratio is found to be

$$
I=\frac{8}{27} \pi k^{4} a^{6}+\frac{40}{81} \pi k^{4} a^{6}=\frac{64}{81} \pi a^{8}(k a)^{4}
$$

approximately. This gives the usual law of intensity varying as the inverse fourth power of the wave-length.

\section{Elastic Spherical Obstacle.}

10. In the case of an elastic incompressible sphere of density $\rho_{0}$ and rigidity $\mu_{0}$ embedded in an incompressible medium of density $\rho$ and rigidity $\mu$, we may write

$$
c_{0}^{2}=\mu_{0} / \rho_{0}
$$

and, since the time-factor $\left(e^{i k c t}\right)$ is every where the same,

$$
k_{0} c_{0}=k c,
$$

whence

$$
\mu_{0} k_{0}^{2}: \mu k^{2}=\rho_{0}: \rho
$$

'The formulw appropriate to the interior of the sphere will be of the same character as those of $\S 1$, with $k_{0}$ written for $k$, and $\mu_{0}$ for $\mu$. 'The typical expressions for the displacements inside the sphere may 
144 Prof. Horace Lamb on the Impact of Waves on a [Mar. 8, therefore be taken to be

$$
\begin{aligned}
& u_{n}^{\prime \prime}=\frac{d}{d x} r^{n} S_{n}^{\prime \prime}+(n+1) \psi_{n-1}\left(k_{0} r\right) \stackrel{d}{d x} \dot{r}^{n \prime} T_{n}^{\prime \prime}-n k_{n}^{2} 2^{2 n+3} \psi_{n+1}\left(k_{0} r\right) \frac{d}{d x} \frac{l_{n}^{\prime \prime \prime}}{r^{n+1}} \\
& +\psi_{n}\left(k_{0} v\right)\left(y \frac{d}{d z}-z \frac{d}{d y}\right) r^{n} U_{n}^{\prime \prime} \\
& \imath_{n}^{\prime \prime}=\frac{d}{d y} r^{n} S_{n}^{\prime \prime}+(n+1) \psi_{n-1}\left(k_{0} r\right) \frac{d}{d y} r^{n} T_{n}^{\prime \prime}-n k_{0}^{2} r^{n+3} \psi_{n+1}\left(k_{0} r\right) \frac{d}{d y} \cdot \frac{T_{n}^{\prime \prime}}{r^{n+1}} \\
& +\psi_{n}\left(k_{0} v\right)\left(z \frac{d}{d x}-x \frac{d}{d z}\right) \cdot r^{n} U_{n}^{n} \\
& w_{n}^{\prime \prime}=\frac{d}{d z} r^{n} S_{n}^{\prime \prime}+(n+1) \psi_{n-1}\left(k_{0} r\right) \frac{d}{d z} r^{n} \dot{T}_{n}^{\prime \prime \prime}-n k_{n}^{2} r^{2 n+3} \psi_{n+1}\left(k_{0} r\right) \frac{d}{d z} \frac{q_{n}^{\prime \prime}}{r^{n+1}} \\
& \left.+\psi_{n}\left(k_{0} v\right)\left(x \frac{d}{d y}-y \frac{d}{d x}\right) r^{n} U_{n}^{\prime \prime}\right)
\end{aligned}
$$

'L'he corresponding stresses $F_{n}^{\prime \prime}, G_{n}^{\prime \prime}, H_{n}^{\prime \prime}$ on a sphere of radius $r$ will be as in (28), with $\mu_{0}$ written for $\mu, k_{0}$ for $k$, and $S_{n}^{\prime \prime}, T_{n}^{\prime \prime}, U_{n}^{\prime \prime}$ for $S_{n}, T_{n}, U_{n}$, respectively.

The continuity of displacement and stress at the surface $r=a$ requires

$$
\begin{aligned}
& u_{n}+u_{n}^{\prime}=u_{n}^{\prime \prime}, \quad v_{n}+v_{n}^{\prime}=i_{n}^{\prime \prime}, \quad w_{n}+w_{n}^{\prime}=w_{n}^{\prime \prime}, \\
& F_{n}+F_{n}^{\prime \prime}=F_{n}^{\prime \prime}, \quad G_{n}+G_{n}^{\prime}=G_{n}^{\prime \prime}, \quad H_{n}+H_{n}^{\prime}=H_{n}^{\prime \prime} .
\end{aligned}
$$

These lead in the usual manner to the formuln

$$
\begin{aligned}
& S_{n}+(n+1) \psi_{n-1}(k a) T_{n}+(n+1) f_{n-1}(k a) T_{n}^{\prime}=S_{n}^{\prime \prime}+(n+1) \psi_{n-1}\left(k_{0}{ }^{\prime}\right) ' I_{n}^{\prime \prime \prime} \text {, } \\
& -n k^{2} a^{2} \psi_{n+1}(k a) T_{n}+\frac{S_{n}^{n}}{a^{2 n+1}}-n k^{2} a^{8} f_{n+1}(k a) I_{n}^{\prime \prime}=-n k_{0}^{2} a^{8} \psi_{n+1}\left(k_{0} a\right) I_{n}^{\prime \prime \prime} \\
& \psi_{n}(k a) U_{n}+f_{n}(k a) U_{n}^{\prime}=\psi_{n}\left(k_{0}(\imath) U_{, n}^{\prime \prime},\right. \\
& \mu A_{n}(k a) S_{n}+\mu C_{n}(k a) T_{n}+\mu A_{n}^{\prime}(k a) \frac{S_{n}^{\prime \prime}}{a^{2 n+1}}+\mu C_{n}^{\prime}(k a) T_{n}^{\prime} \\
& =\mu_{0} A_{n}\left(k_{0} a\right) S_{n}^{\prime \prime}+\mu_{0} C_{n}\left(k_{0} a\right) T_{n}^{\prime \prime} \text {, } \\
& \mu B_{n}(k a) S_{n}+\mu D_{n}(k a) \cdot T_{n}+\mu B_{n}^{\prime}(k a) \frac{S_{n}^{\prime}}{a^{2 n+1}}+\mu D_{n}^{\prime}(k a) T_{n}^{\prime} \\
& =\mu_{0} B_{n}\left(k_{0} a\right) S_{n}^{\prime \prime}+\mu_{0} D_{n}\left(k_{0} a\right) T_{n}^{\prime \prime}, \\
& \mu E_{n}(k a) U_{n}+\mu E_{n}^{\prime}(k a) U_{n}^{\prime}=\mu_{0} E_{n}\left(k_{0} a\right) U_{n}^{\prime \prime} \text {, }
\end{aligned}
$$


where the functions $A_{n}(k a), A_{n}^{\prime}(k a), \& c .$, have the meanings assigned in (29), (30), (31), and (37), (38), (39).

When the extraneous disturbance consists of plane waves, we have $S_{n}=0$. If, further, $k a$ and $k_{0} a$ be both small, the values of $T_{n}^{\prime}$ and $U_{n}^{\prime}$ in terms of $T_{n}^{\prime}$ and $U_{n}$ respectively may be approximated to without much difficulty. Thus, for $n=1$, we find, making use of the formulæ (145) ... (148),

$$
\begin{gathered}
2 T_{1}+\frac{2}{k a} T_{1}^{\prime}=S_{1}^{\prime \prime}+2 T_{1}^{\prime \prime} \\
-\frac{1}{15} k^{2} a^{2} T_{1}+\frac{S_{1}^{\prime}}{a^{3}}-\frac{3}{k^{3} a^{3}} T_{1}^{\prime}=-\frac{1}{15} k_{0}^{2} a^{2} T_{1}^{\prime \prime} \\
-\frac{2}{3} \mu k^{2} a^{\prime \prime} T_{1}-\frac{1}{3} \mu k^{2} a^{2} \frac{S_{1}^{\prime}}{a^{8}}-\frac{2 \mu}{k a} T_{1}^{\prime}=-\frac{1}{3} \mu_{0} k_{0}^{2} a^{2} S_{1}^{\prime \prime}-\frac{2}{3} \mu_{0} k_{0}^{2} a^{2} T_{1}^{\prime \prime}, \\
\frac{1}{15} \mu k^{2} a^{2} T_{1}-6 \mu \frac{S_{1}^{\prime}}{a^{3}}+\frac{18 \mu}{k^{3} a^{3}} T_{1}=\frac{1}{3} \mu_{0} k_{0}^{2} a^{2} S_{1}^{\prime \prime}+\frac{1}{15} \mu_{0} k_{0}^{2} a^{3} T_{1}^{\prime \prime}
\end{gathered}
$$

where only the most important part of each coefficient has been retained.

The solution of these is facilitated by the consideration that $T_{1}$ must be of the order $k^{3} a^{3}$ compared with $T_{1}$, as is seen from the re: sults of $\S 9$, which deals with a particular case of the present problem. Moreover ' $T_{1}^{\prime \prime}$ must be of the same order as $T_{1}$. We thence find, from (165) and (166),

$$
\begin{gathered}
S_{1}^{\prime \prime}+2 T_{1}^{\prime \prime}=2 T_{1}, \\
S_{1}^{\prime \prime} / a^{3}=3 / k^{3} a^{3} . T_{1}^{\prime} .
\end{gathered}
$$

'I'hen (167) leads to the result

$$
{ }^{\prime} T_{1}^{\prime}=\frac{2}{9} k a \frac{\mu_{0} k_{0}^{2} a^{2}-\mu k^{2} a^{2}}{\mu} T_{1}=\frac{2}{9} k^{s} a^{3}\left(\frac{\rho_{0}}{\rho}-1\right) T_{1}^{\prime},
$$

by (155). This agrees with (149) if we put $\rho_{0}=0 . *$

Similarly, the approximate forms of $(16 \mathrm{l})$ and (164) are

$$
\begin{gathered}
\frac{1}{3} U_{1}+\frac{1}{k^{3} a^{3}} U_{1}^{\prime}=\frac{1}{8} U_{.1}^{\prime \prime}, \\
-\frac{1}{1^{5}} \mu l_{i}^{2} a^{2} U_{1}-\frac{3 \mu}{k^{3} a^{3}} U_{1}=-\frac{1}{1^{5}} \mu_{0} k_{0}^{2} a^{2} U_{1}^{\prime \prime},
\end{gathered}
$$

- It is to be noticed that the equations (167) and (168) cannot be assumed to be kufficiently accurate for the deternination of $S_{1}^{\prime \prime}$ and $T_{1}^{\prime \prime}$, owing to the approximnte cancelling of the second and third terms in (168). The main intercst lies, however, in the determination of $\eta_{i}^{\prime}$.

vol.. XxxII.-NO. 719. 
leading to

$$
U_{\mathrm{i}}^{\prime}=\frac{1}{45} k^{5} a^{5}\left(\frac{\rho_{0}}{\rho}-1\right) U_{1},
$$

which also may be compared with (149).

It appears, on reference to (99), that the values of $T_{1}^{\prime}$ and $U_{1}^{\prime}$ are, to our present degree of approximation, the $\operatorname{same}$ as if the sphere were absolutely rigid. This might have been foreseen, for the terms in $T_{1}^{\prime \prime}$ represent, when $k_{0} a$ is small, a mere translation of the sphere as a whole, whilst those in $U_{1}^{\prime \prime}$ represent a pure rotation.

For $n>1$, I find

$$
\begin{aligned}
& T_{n}^{\prime}=-\frac{2\left(n^{2}-1\right)\left(\mu_{0}-\mu\right)}{2\left(n^{3}-1\right) \mu_{0}+\left(2 n^{2}+1\right) \mu} \cdot \frac{(k a)^{2 n-1}}{\{1.3 \ldots(2 n-3)\}^{2}(2 n-1)} T_{n}, \\
& U_{n}^{\prime}=-\frac{(n-1)\left(\mu_{0}-\mu\right)}{(n-1) \mu_{0}+(n+2) \mu} \cdot \frac{(k a)^{2 n+1}}{\{1.3 \ldots(2 n-1)\}^{2}(2 n+1)} U_{n} .
\end{aligned}
$$

These coincide with (143), (144), if we put $\mu_{0}=0$. In particular

$$
T_{2}^{\prime}=-\frac{2\left(\mu_{n}-\mu\right)}{6 \mu_{0}+9 \mu} k^{s} a^{s} . T_{3}, \quad U_{2}^{\prime}=-\frac{\mu_{n}-\mu}{45\left(\mu_{0}+4 \mu\right)} k^{5} a^{5} L_{2} \cdot *
$$

The most important part of the disturbance at a distance in the scattered waves is therefore obtained by writing

$$
B_{1}^{\prime}=\frac{2}{9}\left(\frac{\rho_{0}}{\rho}-1\right) k^{s} a^{\mathrm{y}}, \quad B_{2}^{\prime}=-\frac{2\left(\mu_{0}-\mu\right)}{6 \mu_{0}+9 \mu} k^{s} a^{\mathrm{s}},
$$

in the formulæ (43) and (47), respectively. The approximate value of the dissipation-ratio is

$$
I=\left\{\frac{8}{27}\left(\frac{\rho_{0}}{\rho}-1\right)^{2}+\frac{40\left(\mu_{0}-\mu\right)^{2}}{\left(6 \mu_{0}+9 \mu\right)^{2}}\right\} \pi a^{2}(k a)^{4} .
$$

\section{Effect of Synchronism.}

11. In the various problems above considered, general formulwo have been obtained for the disturbance produced in a train of plane waves by a spherical obstacle constituted in various ways; but no

- When $\left(\rho_{0}-\rho\right) / \rho_{0}$ and $\left(\mu_{0}-\mu\right) / \mu$ are small, we have

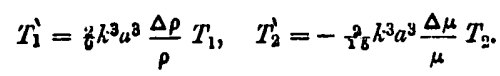

If we substitute the values of $T_{1}$ and $T_{2}$ from (25) and (26), and work out the expressions for the component rotations of the medium, in the scattered wave, we roproduce results given by Lord Rayleigh in a paper already cited (p. 120). 
special examination hus been made of cases where there is coincidence, or approximate coincidence, between the period of the waves and a natural period of vibration of the sphere. In this respect interest attaches chiefly to cases where the free period in question corresponds to a wave-length large in comparison with the circumference of the sphere, but our results will not be altogether dependent on this supposition.

The first instance where a free period of this character was met with was in the rectilinear vibrations of a rigid but movable sphere (§6) whose inertia bears a large ratio $(x)$ to that of an equal volume of the surrounding medium. If in (91) we put $S_{1}=0$, the accurate formula for the scattered waves of type $T_{1}^{\prime}$ takes the form

$$
\frac{T_{1}^{\prime}}{T_{1}}=-\frac{(2 k+1) \psi_{0}(k a)-9 \psi_{1}(k a)}{(2 k+1) f_{0}(k a)-9 f_{1}(k a)}=-\frac{g(k a)}{G(k a)-i g(k a)}
$$

where

$g(k a)=(2 \kappa+1) \psi_{0}(k a)-9 \psi_{1}(k a), \theta(k a)=(2 \kappa+1) \Psi_{0}(k a)-9 \Psi_{1}(k a)$.

The nodulus of the expression in the last member of (180) is never greater than unity; but it attains the value unity, and the amplitude of the scattered waves is therefore a maximum, when

$$
G(k a)=0 .
$$

If we substitute the values of $\Psi_{0}(k a), \Psi_{1}(k a)$ from (4), this equation reduces to

$$
\tan k a=\frac{2 k+1}{9} k a-\frac{1}{k a} \text {. }
$$

The situation of the roots is easily found by a graphical construction, and it appears that when $x$ is large there is a small root given (to a first approximation) by

$$
k a=\frac{3}{\sqrt{ }(2 k-8)} ;
$$

a result which may be compared with (95). When (182) is satisfied exactly, we have

$$
T_{1}^{\prime}=-i T_{1} \text {. }
$$

Hence, putting $B_{1}^{\prime}=-i$ in (64), we have, for the dissipation-ratio,

$$
I=\frac{6 \pi}{k^{2}}=\frac{3}{2 \pi} \cdot \lambda^{2}
$$

where $\lambda$ is the wave-length. 
148 Prof. Horace Lamb on the Impact of Wrves on a [Mar. 8,

A similar argument applies to the rotational vibrations. We find, from (92),

where

$$
\dot{U}_{1}^{\prime}=-\frac{g(k a)}{G(k a)-i g(k a)}
$$

$$
g(k a)=\frac{1}{5} \nu k a \psi_{1}(k a)+\psi_{1}^{\prime}(k a), \quad Q(k a)=\frac{1}{5} \nu k a \Psi_{1}(k a)+\Psi_{1}^{\prime}(k a) .
$$

The wave-length of maximum dissipation is determined, as before, by $G(k a)=0$, or

$$
\tan k a=-\frac{1}{l a a}-\frac{5 k a}{\nu k^{2} a^{2}-15}
$$

The dissipation-ratio, when this is exactly satisfied, is given again by (186).

It is evident that the form of $(180)$ or (187) is quite independent of the special hypotheses here made, or of the value of $n$. The genernl value of the dissipation-ratio $I$, when this is a maximum, is

$$
I=\frac{2 n+1}{2 \pi} \lambda^{2}
$$

where $n$ is the order of the spherical harmonic involved.

The wave-lengths of maximum scattering are in all cuses sharply defined. It would be easy to institute calculations showing the effect of a slight deviation from a critical period; but this point has perlaps been sufficiently illustrated in the former paper."

12. The particular example, just referred to, of a rigid sphere embedded in the medium is in one respect unfitted for the illustration of optical phenomena. In order to obtain a free period large comjared with $a / c$, it was necessary to ascribe to the sphere a great relative inertia. It appear's from $\S 5$ that this implies a very considerable general scattering of incident waves, independently of any epproximation to the critical wave-length. The dissipation, as given ly (74), is, in fact, of the order $(\lambda / a)^{4}$ compared with what we know to be consistent with the actual degree of transparency of the utmosphere. $\dagger$

The difficulty may be met, though perhaps rather artificially, if we have recour'se to the more general suppositions of $\S 7$. Let us

- Proce. Lond. Mrnth. Sori., Vol. xxxir., p. 18.

$\dagger$ Lord Rayleigh, Phil. Mug., April, 1899. 
suppose, for simplicity, that we have a rigid massless shell enclosing a nuclens attached to it by springs. Putting $\kappa=0$ in (106), we have

$$
x^{\prime}=\frac{\kappa_{0} \sigma_{0}^{2}}{\sigma_{0}^{2}-k^{2} c^{2}} \text {. }
$$

If we substitute this for $\mathrm{s}$ in (94), we obtain the equation to determine the free periods, viz.,

$$
\left(1+\frac{2 \kappa_{0} \sigma_{0}^{2}}{\sigma_{0}^{2}-k^{2} c^{2}}\right) k^{2} a^{2}-9 i k a-9=0 .
$$

If the stiffness of the springs and the mass of the nucleus be so adjusted that $\sigma_{0} a / c$ is small, this will be satisfied approximately by

$$
k^{2} a^{2}=\frac{\sigma_{0}^{2} a^{2}}{c^{2}} \div\left(1+\frac{2}{9} \stackrel{\kappa_{0}^{*} \sigma_{0}^{2} a^{2}}{c^{2}}\right) . *
$$

The wave-length of this free period is large compared with $a$, independently of the value of $\kappa_{0}$.

To determine the forced oscillations we find from (180), with $\kappa^{\prime}$ written for $k$, and from (193),

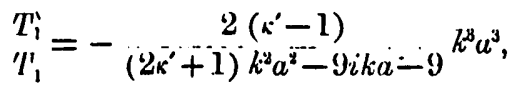

provided the value of $k a$ does not approximate to that given by (19:3). The dissipation-12atio will then be of the usual form

$$
I=C a^{2}(k a)^{4},
$$

where $O$ is a moderate numerical factor.

[Again, we might emplny the hypothesis of an elastic sphere, considered in $\$ 10$. To obtain fiee periods with wave-lengths largo compared with $a$, we must suppose the constants so adjusted that $c_{0}$, defined by (153), is small compared with $c$; in other words, wo must have $\mu_{0} / \rho_{0}$ small compared with $\mu / \rho$. Since we are precluded, for the reason above given, from assigning any grent relative inertia to the sphere, the only assumption left to us is to imagine that $\mu_{0}$ is small compred witb $\mu$.

With molecules adjusted in this manner we might build up a model of a gaseous assemblage freely tirnsparent to racliation

- This indicates that the frecpuency of vibration of the nucleus is less than if the containing shell were held fixed, in the ratio $\left(1+\frac{2 k_{0}{ }^{\kappa_{0}} \sigma_{0}^{2} a^{2}}{c^{2} \cdot .}\right)^{-1}$ 
generally, but with strong absorption for definite wave-lengths. It is to be noticed, however, that in reconciling these requirements we have made it impossible to get rid of that constituent of the general scattered light which is dependent on the harmonic $T_{2}^{\prime \prime}$ and is represented by the formulæ (47).*. If we survey the various problems of this paper, we find that this constituent is always important (with an amplitude proportional to $k^{8} a^{8} / r$ ) except in the case of $\S 10$, where it can be annulled (approximately) by muking $\mu_{0}=\mu$. This is, however, inadmissible for the reason just explained.] $†$

Thursda!, April 5th, 1900.

Lt.-Col. A. J. C. CUNNINGHAM, R.E., Vice-President, in the Chair.

Nineteen members present.

Mr. F. W. B. Frunkland, B.A., Fellow of Clare College, Cambridge, was elected a member.

The following persons were admitted into the Society :-Miss B. M. Cave Browne Cave, Miss F. E. Cave Browne Cave, and Mr. R. W. H. T. Hudson.

Mr. Tucker read a letter from Mrs. J. J. Walker, thanking the Society for its vote of condolence with her on the loss of her husband.

Mr. Macdonald read a note "On the Addition-Theorem for the Bessel Functions," and Mr. Basset made a communication on "The Orthoptic Loci of Curves of a given class."

Prof. Love gave a proof of "The. Uniform Convergence of F'ourier's Series."

Major MacMahon spoke on "An Extension of Orthogonal and Boolian Covariants."

- For the argument that this constituent is absent from the light scattered by finoly divided partiolen, see Lord Rayleigh, Scientific Paper's, Vol. I., p. 107.

+ Added Sept., 1900. 
generally, but with strong absorption for definite wave-lengths. It is to be noticed, however, that in reconciling these requirements we have made it impossible to get rid of that constituent of the general scattered light which is dependent on the harmonic $T_{2}^{\prime \prime}$ and is represented by the formulæ (47).*. If we survey the various problems of this paper, we find that this constituent is always important (with an amplitude proportional to $k^{8} a^{8} / r$ ) except in the case of $\S 10$, where it can be annulled (approximately) by muking $\mu_{0}=\mu$. This is, however, inadmissible for the reason just explained.] $†$

Thursda!, April 5th, 1900.

Lt.-Col. A. J. C. CUNNINGHAM, R.E., Vice-President, in the Chair.

Nineteen members present.

Mr. F. W. B. Frunkland, B.A., Fellow of Clare College, Cambridge, was elected a member.

The following persons were admitted into the Society :-Miss B. M. Cave Browne Cave, Miss F. E. Cave Browne Cave, and Mr. R. W. H. T. Hudson.

Mr. Tucker read a letter from Mrs. J. J. Walker, thanking the Society for its vote of condolence with her on the loss of her husband.

Mr. Macdonald read a note "On the Addition-Theorem for the Bessel Functions," and Mr. Basset made a communication on "The Orthoptic Loci of Curves of a given class."

Prof. Love gave a proof of "The. Uniform Convergence of F'ourier's Series."

Major MacMahon spoke on "An Extension of Orthogonal and Boolian Covariants."

- For the argument that this constituent is absent from the light scattered by finoly divided partiolen, see Lord Rayleigh, Scientific Paper's, Vol. I., p. 107.

+ Added Sept., 1900. 
Messrs. Macaulay, R. W. H. T. Hudson, Whittaker, and the Chairman spoke to one or more of the papers.

A paper by Mr. Bromwich, entitled "Note on Weierstrass's Reduction of a Family of Bilinear Forms," was received.

The Chairman announced that the May meeting would be made "special," as announced by letter to the member's of the Society.

The following presents were made to the Library :-

" Educational Times," April, 1900.

“Indian Engineering," Vol. xxvir, No8. 7-10, Feb. 17-Mar. 10, 1900.

“Nautical Almanac for 1903," 8vo ; Edinburgh, 1900. From Nautioal Almanac Office.

"Mathematical Gazette," Vol. I., No. 20 ; 1900.

"Periodico di Matematica," Serie 2. Vol. n., Faso. 5 ; Livorno, 1900.

"Supplemento al Periodico di Matematica," Anno Irr., Fasc. 5; Livorno, 1900.

Darboux, G._"Sur la Déformation des Surfaces du second degre," 4to ; Paria. 1900.

Ontwald, W.- "Perindische Erscheinungen bei der Auflösung des Chrome in Siiuren," 8vo ; Leipzig, 1900.

Rice, H._-"The Theory and Practice of Interpolation," 8vo ; Lynn, Mass., 1900. From the author.

"Trnnsactions of the American Mathematical Society," Vol. r., No. 1; 1900.

"Memoirs of the National Academy of Sciences," Vol. vir., Memoir 4; Washingtou, 1899.

"Grundlage der Geometrie" (3 pp.), and "Euklidische Grundlage der Geometrie" (7 pp.), from the author, Dr. W. Veltmann.

"Report on the Progress of the Solution of the Problem of Three Bodies," by E. T. Whittaker (reprint from " British Association Report," 1899).

The following exchanges were received :-

"Proceedings of the Royal Society," Vol. Lxvr., Nos. 426, 427 ; 1900.

"Beibliitter zu den Annalen der Physik und Chemie," Bd. xxrv., St. 2, 3; Leipzig, 1900.

"Annales de la Faculté des Sciences de Toulouse," Série 2, Tome 1., 3me Fascicule; 1899.

"Bulletin of the American Mathematical Society," Series 2, Vol. vi.; No8. 5, 6, Feb.-Mar., 1900; New York.

"Bulletin des Sciences Mathématiques," Tome xxrv., Jan., 1900 ; Paris.

"Rendiconto dell' Accademia delle Scienze Fisiche e Matematiche," Sorie 3, Vol. vi., Nos. 1, 2: Nupoli, 1900.

"Atti della Reale Accademia dei Lincei-Rendiconti," Sem. 1, Vol. Ix., Fasc. 4, 5 ; Roma, 1900.

"Nyt Tidsskrift for Matemntik," B. Aargang xr., Nr. 1 ; Copenhagen, 1900.

" Sitzungrberichte der Königl. Preuss. Akademio der Wissenschaften zu Berlin," Nos. 46,47 ; Nov., 1897. 